\title{
Finite-size modifications of the magnetic properties of clusters
}

\author{
Hendriksen, Peter Vang; Linderoth, Søren; Lindgård, Per-Anker
}

Published in:

Physical Review B

Link to article, DOI:

10.1103/PhysRevB.48.7259

Publication date:

1993

Document Version

Publisher's PDF, also known as Version of record

Link back to DTU Orbit

Citation (APA):

Hendriksen, P. V., Linderoth, S., \& Lindgård, P-A. (1993). Finite-size modifications of the magnetic properties of clusters. Physical Review B, 48(10), 7259-7273. https://doi.org/10.1103/PhysRevB.48.7259

\section{General rights}

Copyright and moral rights for the publications made accessible in the public portal are retained by the authors and/or other copyright owners and it is a condition of accessing publications that users recognise and abide by the legal requirements associated with these rights.

- Users may download and print one copy of any publication from the public portal for the purpose of private study or research.

- You may not further distribute the material or use it for any profit-making activity or commercial gain

- You may freely distribute the URL identifying the publication in the public portal 


\title{
Finite-size modifications of the magnetic properties of clusters
}

\author{
P. V. Hendriksen* and S. Linderoth* \\ Laboratory of Applied Physics, Technical University of Denmark, DK-280o Lyngby, Denmark \\ P.-A. Lindgård \\ Physics Department, Risø National Laboratory, DK-4000 Roskilde, Denmark
}

(Received 1 March 1993)

\begin{abstract}
The spin-wave spectrum of Heisenberg spin clusters of various structures (bcc, fcc, and disordered) ranging in size between 9 and 749 spins is calculated by a self-consistent diagonalization of the equation of motion of $S^{+}$in real space. The spin-wave spectrum of the clusters is strongly modified relative to the bulk, and the consequent neutron-scattering cross section exhibits discretely spaced wave-vector-broadened eigenstates. The implications of the finite size on thermodynamic properties, like the temperature dependence of the magnetization and the critical temperature, are also elucidated. We find the temperature dependence of the cluster magnetization to be well described by an effective power law, $M_{\text {mean }} \propto 1-B T^{\alpha}$, with a size-dependent, but structure-independent, exponent larger than the bulk value. The critical temperature of the clusters is calculated from the spin-wave spectrum by a method based on the correlation theory and the spherical approximation generalized to the case of finite systems. A size-dependent reduction of the critical temperature by up to $50 \%$ for the smallest clusters is found. The trends found for the model clusters are extrapolated to the size regime of nanoscale particles.
\end{abstract}

\section{INTRODUCTION}

The study of the magnetic properties of ultrafine metallic particles and metal clusters is an active field of research. ${ }^{1-7}$ Ultrafine particles and clusters are interesting from a fundamental point of view as they allow the study of how the material properties are affected by the finite size of the system. Nanoscale clusters make the bridge between the bulk system and the atom, and the study of clusters may show how the bulk properties evolve from the atomic properties when increasing the number atoms in the clusters. Also information about the special properties of surfaces can be obtained from studies of clusters and ultrafine particles as in such systems a relatively large fraction of the atoms will be at the surface. Ultrafine magnetic particles are also of technological importance due to their use in magnetic recording media and ferrofluids. ${ }^{8,9}$ (In the following the phrase cluster is used for particles holding on the order of tens to hundreds of atoms - slightly larger particles consisting of thousands to a few $10^{6}$ of atoms, with particle diameters in the range from 1 to $100 \mathrm{~nm}$, are referred to as nanoscale or ultrafine particles.)

Recently, de Heer, Milani, and Châtelain ${ }^{1}$ and Bucher, Douglass, and Bloomfield ${ }^{2}$ succeeded in preparing free iron and cobalt clusters in the size regime of 10 to 500 atoms, thereby bridging the gap between the atom and the finest supported metallic particles produced to date $^{3-5}$ (with particle diameters on the order of 2-3 $\mathrm{nm}$ ). Iron and cobalt can thus be studied over the full size range. Clusters of $\mathrm{Fe}$ and Co order ferromagnetically like the bulk phases. At low temperatures they show enhanced magnetic moments per atom compared to the bulk ${ }^{2,6,7,10}$ due to finite-size effects on the electronic properties. ${ }^{11-13}$ Like ultrafine magnetic particles, they behave superparamagnetically. The magnetization curves of the clusters can be explained on the basis of the theory of superparamagnetic relaxation, assuming that the atomic magnetic moments inside the clusters are parallel..$^{6,7}$

Much theoretical effort has been devoted to the electronic, structural and magnetic ground-state properties of clusters. ${ }^{11-19}$ Intrinsic thermodynamic properties, such as the temperature dependence of the magnetization and the Curie temperature, have been addressed in a number of Monte Carlo model studies of finite-size Heisenberg and Ising spin systems. ${ }^{20-23}$ These studies have shown a "rounding" of the critical behavior ${ }^{20-22}$ and that the magnetization in a finite system is inhomogeneous due to surface effects. ${ }^{20,23}$

Here we address the statistical properties of a single magnetic cluster. We calculate the thermodynamic properties of Heisenberg clusters of various structures and sizes on the basis of a self-consistently solved spin-wave spectrum. This approach allows a prediction of the dynamical behavior of the spin system (which is very difficult in a Monte Carlo study) and the consequent neutronscattering cross section. Deviations from the bulk behavior for the temperature dependence of the magnetization brought about by the finite size of the clusters are analyzed. By a generalization of the spherical approximation the effective critical temperature $T_{c}$ of the clusters is calculated. $T_{c}$ is defined by a maximum susceptibility criterion. Calculations are performed for clusters in the size range from 9 to 749 spins. A good estimate of the behavior of larger clusters and ultrafine particles can be made by extrapolating the trends found for the small clusters (9-749 spins) to known bulk behavior.

In a previous study ${ }^{24}$ we addressed the size dependence of the magnetic properties of bcc-structured clusters by 
the above-described method. In Ref. 24 we concentrated on the size dependence of the temperature dependence of the magnetization, and we compared the predictions of the model with experimental studies of ultrafine iron particles reported in the literature. Here we vary the structure of the clusters (fcc, bcc, and disordered) and we address the dependence on both size and structure of the thermodynamic properties. Moreover, results of a method of calculating the critical temperature for clusters are reported.

The results of the model calculations are compared to recent experimental $\mathrm{data}^{25}$ on the temperature dependence of the magnetization of ultrafine, supported iron particles.

\section{THEORY}

The Heisenberg model Hamiltonian is given by

$$
H=-\frac{1}{2} \sum_{i j} J_{i j} \mathbf{S}_{i} \cdot \mathbf{S}_{j}
$$

where $J_{i j}$ are the exchange energy constants and $\mathbf{S}_{i}$ and $\mathbf{S}_{j}$ are the spins on sites $i$ and $j$. For simplicity only nearest-neighbor interactions $\left(J_{i j}=J>0\right)$ are taken into account, that is, $J_{i j}=0$ when all but neighboring spins are considered. In the ground state all spins are parallel, defining the $z$ direction. To find the eigenstates of the spin system with this Hamiltonian the problem is best phrased in terms of the raising and lowering operators $S^{+}=S_{x}+i S_{y}$ and $S^{-}=S_{x}-i S_{y}$, as this will result in a single equation of motion in $S^{+}$and $S_{z}$ instead of two coupled equations in $S_{x}, S_{y}$, and $S_{z}$. The quantum-mechanical equation of motion describing the propagation of a spin deviation $\left(i \hbar \frac{d S_{i}^{+}(t)}{d t}=\left[S_{i}^{+}(t), H\right]\right)$ can in a (symmetrical) site-dependent random-phase approximation be written

$$
\hbar \omega S_{i}^{+}=\sum_{j} A_{i j} S_{j}^{+}
$$

where

$$
A_{i i}=\sum_{j} J_{i j} M_{j}, \quad A_{i j}=-J_{i j} \frac{M_{i}+M_{j}}{2} .
$$

$M_{i}=\left\langle S_{z i}\right\rangle$ is the thermally averaged mean value of the spin at site $i$, and $\omega$ is the angular frequency introduced by assuming $S^{+}(t)=S^{+} \exp (i \omega t)$. In Eq. (3) an approximation that symmetrizes Eq. (2) has been made in addition to the random-phase approximation. In general, the equation of motion of $S^{+}$is asymmetric in a site-dependent random-phase approximation, as the offdiagonal terms in Eq. (2) are proportional to $M_{j}$, which in a finite system must be expected to vary from site to site. The eigenvalues of Eq. (2) will thus be complex, which means that the eigenstates are damped spin waves. In the case of a finite system, damping thus appears already within the random-phase approximation, unlike in the bulk case. As we do not want to treat this complicating effect on the present level of sophistication, we symmetrize the equation of motion by the approximation

$$
M_{j} \approx\left(M_{i}+M_{j}\right) / 2 .
$$

This approximation is of no consequence for calculations of the properties at $T=0$, as in this case $M_{j}=S$ for all spins. As $M_{j}$ varies only slowly across the clusters in the temperature range in which we want to solve the problem, Eq. (4) represents a good approximation.

The equation of motion in $S^{-}$is identical to Eq. (2) with $S^{-}(t)=S^{-} \exp (-i \omega t)$, and hence $S_{x}$ and $S_{y}$ can be written

$$
S_{x}=S^{+} \cos (\omega t), \quad S_{y}=S^{+} \sin (\omega t) .
$$

$S^{+}$is thus, in a classical interpretation, the amplitude in the precession movement of the spin (the opening radius of the cone on which the spins precess).

In the bulk $M_{i}$ is the same at all sites due to the translational invariance. In this case Eq. (2) can be diagonalized by a Fourier transformation to the reciprocal $\mathbf{q}$ space. The eigenstates are plane waves with a dispersion given by ${ }^{26}$

$$
\hbar \omega(\mathbf{q})=S\left(J_{\mathbf{0}}-J_{\mathbf{q}}\right)
$$

where

$$
J_{\mathbf{q}}=\sum_{i} J_{\mathbf{a}_{\mathbf{i}}} e^{-i \mathbf{q} \cdot \mathbf{a}_{\mathbf{i}}}
$$

$\mathbf{a}_{\mathbf{i}}$ are the vectors joining a spin to its nearest neighbors. $J_{0}=J_{\mathbf{q}=\mathbf{0}}$ is the exchange energy constant times the coordination number (number of nearest neighbors). [In the ordered clusters we adopt the simplifying assumption that all nearest-neighbor exchange energy constants are equal $\left(J_{\mathbf{a}_{\mathbf{i}}}=J\right)$. However, $J_{\mathbf{a}_{\mathbf{i}}}$ is allowed to vary in the disordered clusters.] In the case of a finite system a Fourier transformation will no longer diagonalize Eq. (2) as the translational invariance is lost; $\mathbf{q}$ is no longer a good quantum number. The matrix eigenvalue problem [Eq. (2)] can, however, easily be solved numerically in real space. The diagonalization of Eq. (2) for a system consisting of $N$ spins yields $N$ eigenvalues $\left(E_{p}, p=0,1, \ldots, N-1\right)$ and the corresponding eigenvectors $\left(\psi_{i}^{p}\right)$.

Assuming a fully aligned ferromagnetic ground state at $T=0$ the diagonalization of Eq. (2) is straightforward. In this case $M_{i}=\left\langle S_{i z}\right\rangle=S$, and hence the off-diagonal elements $\left(A_{i j}\right)$ are equal to either $S J$ or zero, and the diagonal terms $\left(A_{i i}\right)$ are equal to the number of nearest neighbors of the considered spin (i) times $S J$. At finite temperatures the problem becomes more involved, since the excitation of spin waves leads to a lowering of the $M_{i}$ values from the maximum value depending on both position and temperature. The magnetization profile $\left(M_{i}\right)$ is calculated by adding the statistically weighted contributions from all excited states contributing to the spin deviation on each site $i$,

$$
M_{i}=S-\sum_{p=1}^{N-1}\left|\psi_{i}^{p}\right|^{2} n_{p}
$$

where $n_{p}=\left[\exp \left(E_{p} / k_{B} T\right)-1\right]^{-1}$ is the Bose weight of the state and $N$ the number of spins. The eigenvectors 
are normalized $\left(\sum_{i}\left|\psi_{i}^{p}\right|^{2}=1\right)$; hence the total spin is lowered by one in each eigenstate. Here, as in the standard treatment of the infinite Heisenberg spin system, it is assumed that a superposition of independent single spin-wave states is a good approximation of the many spin-wave states, in consistency with the random-phase approximation. The coefficients of Eq. (2) now depend on its solution [through Eq. (8)], and hence the problem has to be solved self-consistently.

The neutron-scattering cross section $S(\mathbf{q}, \omega)$ can be calculated from the eigenvectors $\left(\psi_{i}^{p}\right)$ after a Fourier transformation to $\mathbf{q}$ space:

$$
S(\mathbf{q}, \omega)=\mathcal{K} \sum_{p=1}^{N-1}\left|S_{p, \mathbf{q}}^{+}\right|^{2} \delta\left(\hbar \omega-E_{p}\right)
$$

where

$$
S_{p, \mathbf{q}}^{+}=\sum_{i} \psi_{i}^{p} e^{i \mathbf{q} \cdot \mathbf{r}_{i}}
$$

$\mathbf{r}_{\mathbf{i}}$ is the vectorial representation of the cluster, and $\mathcal{K}$ is a constant which is of no interest in the present context.

The lowest eigenvalue of Eq. (2) is $E=0$. This state $\left(\psi_{i}^{0}=N^{-1 / 2}, \mathbf{q}=\mathbf{0}\right)$ plays a special role. It reflects the fact that when no anisotropy energy is included in Eq. (1) and no magnetic field is applied, the ground-state quantization axis is not uniquely defined. The spins are parallel in this state, and the total spin equals $N S$ on a redefined quantization axis. As discussed by Glass and Klein ${ }^{27}$ and later proved by Döring ${ }^{28}$ this state has to be omitted in the summations over excited states, when calculating thermodynamic properties of a finite-size Heisenberg spin system.

The clusters discussed in the following sections have been set up by spherical cutouts from fcc or bcc lattices around a central spin. The clusters thus consist of concentric shells of spins in equivalent sites. The two structures represent extremes in packing density. In order to study the intermediate densities and in order to study the implications of disorder, clusters with holes and disordered structures have also been treated. The holes have been introduced randomly and disorder introduced by a random displacement of the spins relative to an underlying fcc or bcc lattice.

\section{EXCITATION SPECTRUM AT $T=0$}

In this section we consider the implications of finite size on the properties of the Heisenberg spin cluster at zero temperature. In the previous section it was described how the eigenstates of the cluster can be found by solving an eigenvalue problem of $S_{i}^{+}$and the classical interpretation of the eigenstates was discussed. An example of a cluster eigenstate in the upper half of a fcc cluster consisting of 55 spins is given in Fig. 1. This cluster contains five [100] planes. The values of $S_{i}^{+}$(given by the height of the spikes) of the third eigenstate are shown for the central [Fig. 1(a)] and two upper [100] planes [Figs. 1(b) and $1(\mathrm{c})$ ] of the cluster. The eigenvalue is 3 times degenerate with similar states for the [010] and [001] planes.
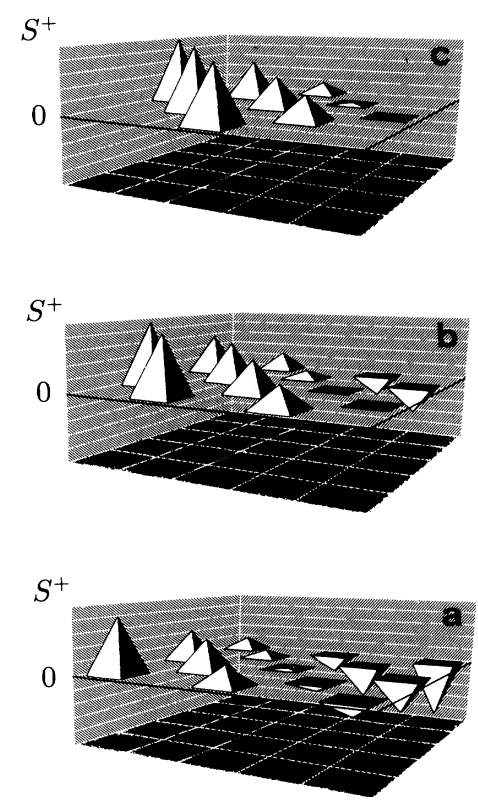

FIG. 1. Third eigenstate of a 55-spin fcc cluster illustrated in real space for the upper half of the cluster. The cluster has been cut along the [100] planes: (a) is the central plane and (b) and (c) the two upper planes. The height of the spikes indicate the magnitude of $S^{+}$, and the direction indicates the sign. A change of sign corresponds to a phase shift in the precession movement of $180^{\circ}$.

The influence of the coordination lacking at the surface is immediately seen; in general, the outer spins have the largest values of $S_{i}^{+}$. The magnitude of $S_{i}^{+}$gives the amplitude of the spin wave or, when considering $S_{i}^{z}$, the lowering relative to the ground-state value $(S)$. It is clear from Fig. 1 that the state is not a simple plane wave and therefore cannot be characterized by just one wave vector q. The change of sign of $S_{i}^{+}$across the central spin, observed in the central plane [Fig. 1(a)], signifies that the spins on either side of the center, classically speaking, are precessing out of phase by $180^{\circ}$ [cf. Eq. (5)]. The depicted state has a node at the central spin, which means that excitation of this specific state will not reduce $S_{i}^{z}$ from the ground-state value at the center. Maximum amplitude is adopted on some of the surface spins. In the depicted state $S_{i}^{+}$varies slowly across the cluster. The higher-lying states are characterized by faster variations in $S_{i}^{+}$.

\section{A. Neutron-scattering cross section}

The finite size of the clusters will lead to modifications of the neutron-scattering cross section $[S(\mathbf{q}, \omega)]$ from that characteristic of the bulk; first, a discrete set of energy values and, second, a broad wave-vector response. This is because the eigenstates can no longer be characterized by a single $\mathbf{q}$ value. In Fig. $2, S(\mathbf{q}, \omega)$, calculated according to Eqs. (9) and (10), is shown at $T=0$ for a number of clusters of different sizes and structures for $\mathbf{q}$ along the [111] direction. $S(\mathbf{q}, \omega)$ is strongly modified compared 


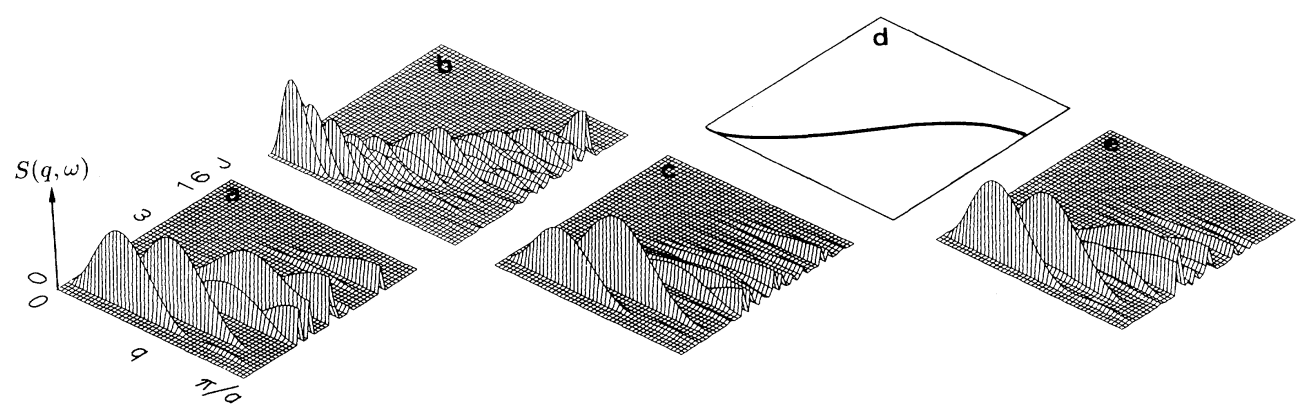

FIG. 2. Neutron-scattering cross section $S(\mathbf{q}, \omega)$, for $\mathbf{q}$ along the [111] direction, for a number of clusters of different size and structure: (a) a fcc cluster containing 55 spins, (b) a fcc cluster containing 683 spins, and (c) a disordered fcc cluster containing 55 spins. The disturbances in (c) are made by random displacements of the atoms by up to $10 \%$ of the cubic unit-cell dimension, imposed on an underlying fcc structure. In (d) the bulk dispersion curve in the [111] direction is shown for comparison. In (e) holes have been randomly introduced at $10 \%$ of the sites in a 55 -atom fcc cluster. $a$ is the unit-cell length.

to bulk behavior. A continuous distribution of $q$ values is needed to describe the spin deviation pattern of the discrete eigenstates. The position of the peak in $S(\mathbf{q}, \omega)$ shifts toward larger $q$ values with increasing energy of the eigenstate. The dominant wavelengths characterizing the eigenstate thus decrease with increasing energy of the eigenstate, as expected from the above discussion of the appearance of the eigenstates in real space. The spread of $q$ values is due to the finite size of the system ( $S=0$ outside the cluster), and as the size of the cluster increases [moving from Fig. 2(a) to 2(b)] the $q$ distribution becomes narrower, approaching the $\delta$-function response and the bulk dispersion curve [Fig. 2(d)]. The limited number of degrees of freedom result in appreciable energy gaps between the eigenvalues. The energy gap between the ground state and the first excited state $(\Delta E)$ is of particular importance. $\Delta E$ is clearly seen in Fig. 2, and it is seen that $\Delta E$ decreases with increasing cluster size [compare Figs. 2(a) and 2(b)]. $S(\mathbf{q}, \omega)$ calculated for $\hat{\mathbf{q}}=\mathbf{q} /|\mathbf{q}|$ along other directions shows a similar pattern. The eigenstates of the cluster bear some resemblance with the bulk q states; for instance, the excited states are generally at lower energies when $\hat{\mathbf{q}} \|[111]$ than when $\hat{\mathbf{q}} \|[100]$. It is therefore convenient to discuss the states in terms of the high-symmetry $\mathbf{q}$ directions. However, as previously discussed, a large number of $\mathbf{q}$ vectors are needed to characterize an eigenstate in the cluster. A manifestation of this can be seen when comparing Figs. 2(b) and 2(d). One might expect that the lacking coordination of the surface spins will result in an overall softening of the eigenstates of the clusters relative to those of the bulk. However, the most energetic state in the [111] direction of the 683 cluster actually lies above the bulk dispersion curve. This shows that it is not a pure $\mathbf{q}=[111]$ state.

In Figs. 2(c) and 2(e) the influence of disorder in the cluster structure on $S(\mathbf{q}, \omega)$ is illustrated. The exchange energy constant $J_{i j}$ can for small disturbances be expanded as $J_{i j}=J\left(r_{0}\right)+\Delta r J^{\prime}\left(r_{0}\right)$, where $r_{0}$ is the nearest-neighbor distance in the fcc lattice and $\Delta r$ the disturbance. In Fig. 2(c) the $J_{i j}$ values have been randomly disturbed by $\pm 20 \%$ with a perturbation of the underlying fcc structure by up to $10 \%$ of the nearest- neighbor distance. The exchange energy constants $J_{i j}$ were, for simplicity, assumed to vary as given above with $J^{\prime}\left(r_{0}\right)=-J\left(r_{0}\right) / r_{0}$. The disturbance of the spin positions alters the exchange energy constants $J_{i j}$ and destroys the symmetry of the clusters. Hence, the degeneracy of the eigenstates is lifted [compare Figs. 2(a) and $2(\mathrm{c})]$. However, $\Delta E$ and the mean spin-wave energy remain roughly unchanged by the disordering of the cluster. The shift of the eigenvalues depends only on the product of $\Delta r$ and $J^{\prime}\left(r_{0}\right)$, and no effect of changing the sign of $J^{\prime}\left(r_{0}\right)$ is found. The eigenfunctions explicitly depend on $\Delta r$. Even assuming an unrealistically strong dependence of $\Delta r\left[J^{\prime}\left(r_{0}\right)=-J\left(r_{0}\right) / r_{0}\right]$ there is no drastic effect on the q dependence of $S(\mathbf{q}, \omega)$ [cf. Fig. 2(c)]. The effect of introducing holes, randomly at $10 \%$ of the spin sites, is illustrated in Fig. 2(e). The holes, like the disturbance of the spin positions, give rise to a lifting of the degeneracy because the symmetry of the cluster is destroyed. The introduction of holes, however, further leads to an overall softening of the states, due to the reduction in the mean number of nearest neighbors.

\section{B. Size dependence of the energy gap}

The energy gap between the ground state and the first excited state $\Delta E$ is important for the thermodynamic properties. As will be shown later the energy gap can be quite substantial and should indeed be experimentally detectable. It is therefore interesting to follow the variation of $\Delta E$ with cluster size. A simple expression for this dependence will be given in the following.

Spin waves in the bulk are most easily excited perpendicular to the most dense planes [cf. Eq. (6)], i.e., for $\hat{\mathbf{q}}$ along the [111] and [110] directions in the fcc and bcc lattices, respectively. The first eigenstate seen along the [111] direction in three fcc clusters of different size is illustrated in Fig. 3. Here the sums of $S_{i}^{+}$in the [111] planes are plotted versus the distance from the center to the planes. In all three clusters the eigenstate has a node in the center. In the smallest cluster the extrema are adopted in the outermost planes. However, the extrema are found to move inward when increasing the cluster 


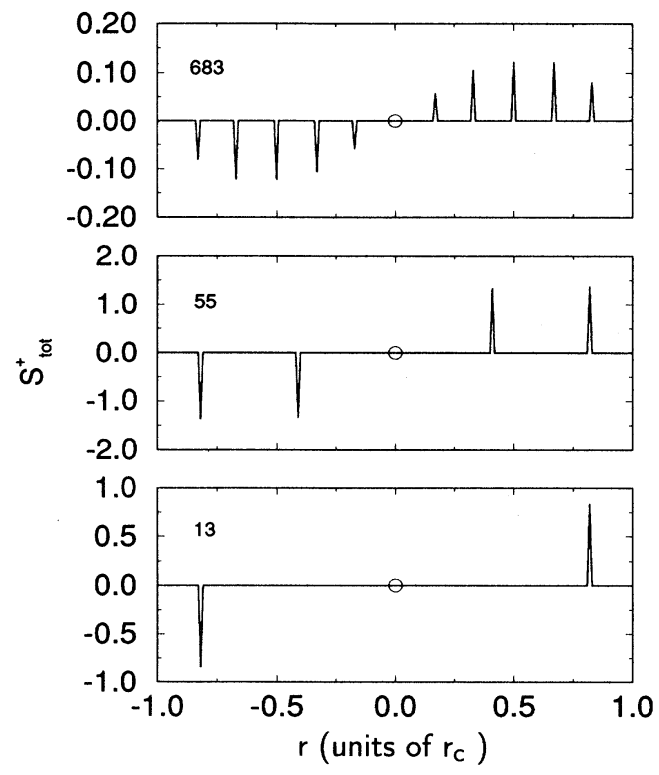

FIG. 3. Projection of the first eigenstate on the [111] direction for fcc clusters consisting of 13,55 , and 683 spins. The heights of the spikes are the sums of $S_{i}^{+}$in the [111] planes and the abscissa the distance from the center to the considered plane.

size. One can interpret the distance between the extrema as a characteristic half wavelength $\lambda$ of the state. The characteristic wavelength thus varies from being equal to twice the cluster dimension for the smallest cluster to become somewhat smaller than twice the cluster dimension for the larger clusters. Now, if we assume (1) that the energy of the eigenstates depends on the square of the characteristic wave number, as for the bulk $\left(\Delta E=J S a^{2} q^{2}\right)$, and (2) that the characteristic half wavelength varies in a simple way from being equal to the cluster dimension $\left(2 r_{c}\right)$ for the smallest cluster $\left(r_{c}=r_{0}\right)$ to become a certain fraction $\xi$ of the dimension in the continuum limit $\left(r_{c} \gg r_{0}\right)$, one arrives at the following expression for the size dependence of $\Delta E$ :

$$
\begin{aligned}
\Delta E\left(r_{c}\right) & =J a^{2} S\left(\frac{2 \pi}{\lambda\left(r_{c}\right)}\right)^{2} \\
& =\frac{J a^{2} S \pi^{2}}{4}\left[\xi r_{c}+(1-\xi) r_{0}\right]^{-2} .
\end{aligned}
$$

Here $\lambda$ was assumed to vary with size according to the expression

$$
\lambda=4 r_{0}\left(\xi \frac{r_{c}}{r_{0}}+(1-\xi)\right),
$$

which fulfills the requirements stated above. $a$ in Eq. (11) is the length of the cubic unit cell. The cluster dimension is expressed in terms of $r_{c}$, defined as the radius of a sphere (that holds the same number of spins as the setup cluster), assumed to have a density equal to that of the bulk lattice (fcc or bcc). This gives a smoothly varying size measure.
The energy gap $\Delta E$ for bcc clusters in the size range from 9 to 749 spins and for fcc clusters in the size range from 13 to 683 spins is plotted as a function of $r_{c}^{-1}$ in Fig. 4. The solid curves in Fig. 4 are the analytical expression of $\Delta E$ given by Eq. (11) with $\xi=0.71 .^{29}$ This value is consistent with that found for the similar eigenvalue problems of the magnetic and electric fields in a continuum sphere. ${ }^{30}$ The wavelength argument leading to Eq. (11) accounts very well for the size dependence of $\Delta E$ for both fcc- and bcc-structured clusters. The energy gap for clusters larger than those for which calculations have been performed can thus be calculated directly from Eq. (11). In the limit of the smallest clusters, it is valid to consider $\Delta E$ to correspond to the maximum wavelength standing waves in the clusters. For larger clusters the cutoff wavelength is about 1.4 times the cluster dimension, in good agreement with the continuum theory. Because of the larger coordination in the fcc lattice than in the bcc lattice, the $\Delta E\left(r_{c}\right)$ curve of the fcc clusters lies above the curve for the bcc clusters.

The energy gaps of the smallest bcc clusters show some scatter around the trend set by the larger clusters and the analytical expression. This is an effect of the detailed structure of the outermost shells in the cluster which can be more or less open. The scatter of the points can be accounted for if one considers an effective coordination number (mean number of nearest neighbors) for the cluster, defined as

$$
z_{\text {eff }}=\mathcal{N}_{s} / N=z-\mathcal{N}_{u} / N
$$

where $\mathcal{N}_{s}$ and $\mathcal{N}_{u}$ are the number of satisfied and unsatisfied "bonds," respectively. $\mathcal{N}_{u} / N$ varies as surface area to volume $\left(\mathcal{N}_{u} / N \propto 1 / r\right)$. The effective coordination number $z_{\text {eff }}$ thus increases with cluster size $r_{c}$, approaching the bulk coordination number $z$ (equal to 12 and 8 for the fcc and bcc lattices, respectively). There is a structural effect superimposed on the size trend. The effect on $z_{\text {eff }}$ of adding one more shell to a cluster depends on the number of spins in the added shell and on the configuration of the outermost shells of the cluster

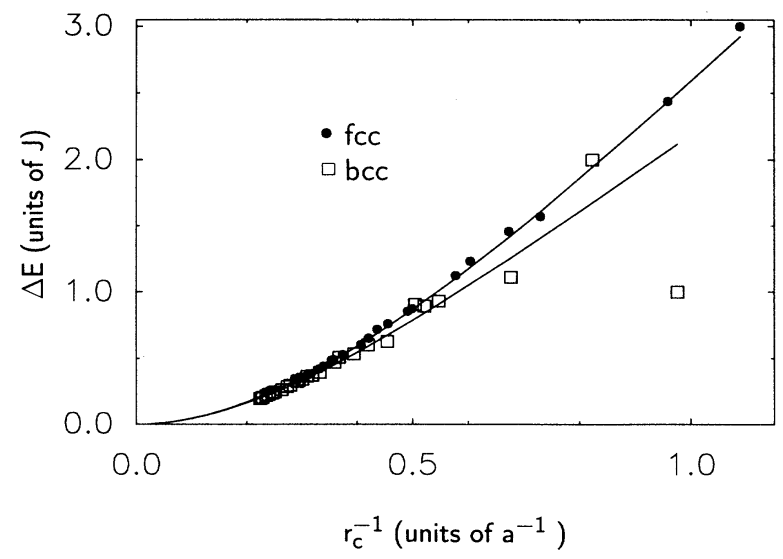

FIG. 4. Energy gap $\Delta E$ in the spin-wave spectrum shown as a function of $1 / r_{c}$ for fcc $(\bullet)$ and bcc $(\square)$ clusters. The solid curves are theoretical curves (see text). 
to which it is added. The variation in the mean number of nearest neighbors $\left(z_{\text {eff }}\right)$ with cluster size is illustrated in Fig. 5. The deviations from the overall trend of the size dependence of the energy gap and the mean number of nearest neighbors are seen to be strongly correlated, when comparing Figs. 4 and 5. Variations in the structure of the outermost shells of the cluster thus account for the deviations from the overall size dependence of the energy gap observed for the smallest bcc clusters. The importance of the structure of the outermost shells of the cluster (the cluster "closure") is in a way an artifact of the way we set up the model clusters. It is important to realize the effect in order to be able to focus on the size trends. In real clusters a reconstruction of the structure toward more close-packed surfaces might take place, partially eliminating the strong structural variations in $z_{\text {eff }}$. To simplify the discussion we neglect this possibility.

The energy gaps in the spin-wave spectrum can be quite large. Assuming that the exchange energy constant in the clusters is of the same order of magnitude as in the bulk $[J=11.9 \mathrm{meV}$ for $\alpha-\mathrm{Fe}$ (Ref. 31)] the energy gaps of a 9- and a 749-spin $\alpha$-iron cluster correspond to about $140 \mathrm{~K}$ and $30 \mathrm{~K}$, respectively.

\section{Density of states}

The thermodynamic properties of the clusters for finite temperatures depend on the full density of states of the spin-wave spectrum. Characteristic measures of the density of states and their size dependence are illustrated in Fig. 6 (at $T=0$ ). A typical example of the density of states is shown as an inset. The energy of the highest-lying eigenvalue increases with increasing cluster size, from $13 J$ and $9 J$ for the 13-spin fcc and 9spin bcc clusters, respectively, to the bulk value of $16 J$ (characteristic of a wave in the [100] direction with a wavelength of twice the lattice constant) in the limit of an infinite system. The effect discussed earlier, where the cluster eigenstate was found to be more energetic than the bulk [111] state (cf. Fig. 2), is only found when a comparison of cluster states to specific bulk states is made. The lack of full coordination in the clusters is re-

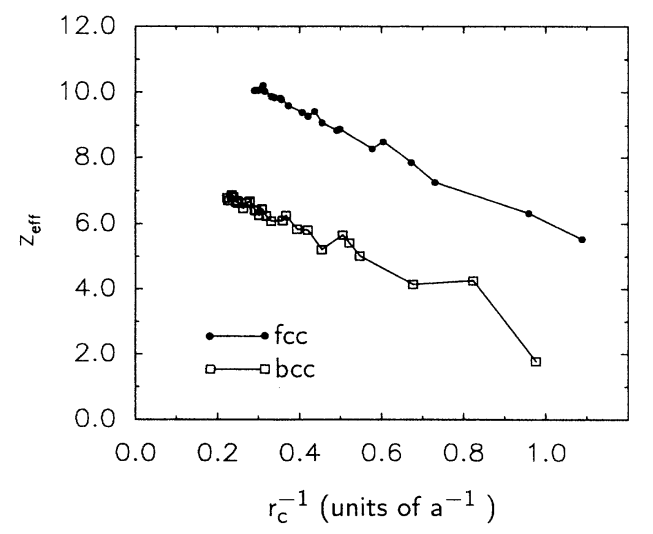

FIG. 5. Cluster-size dependence of the mean number of nearest neighbors $\left(z_{\text {eff }}\right)$ in the cluster [fcc $(\bullet)$, bcc $\left.(\square)\right]$. sponsible for the observed lowering of $E_{\max }$ compared to the bulk value. This surface effect, of course, influences all the eigenvalues. Hence, the mean spin-wave energy is also found to increase with increasing cluster size, slowly approaching the bulk values of $8 J$ and $12 J$ for bcc and fcc clusters, respectively. The mean energy is exactly equal to the effective coordination number $z_{\text {eff }}$ times the exchange energy constant. This follows from the identity $\operatorname{Tr}\left(A_{i j}\right)=\operatorname{Tr}\left(E_{i j}\right)$ in Eq. (2), where $E_{i j}=E_{i} \delta_{i j}$ is the diagonal matrix of the eigenvalues. $\operatorname{Tr}\left(A_{i j}\right) / J S N$ is the mean number of nearest neighbors as the diagonal terms $\left(A_{i i}\right)$ of $A_{i j}$ are the mean fields experienced by the spins and $\operatorname{Tr}\left(E_{i j}\right) / N$ is the mean energy. The standard deviations $(\sigma)$ of the density of states for the fcc and bcc clusters are also shown in Fig. 6. They are effectively independent of size.

An approximate evaluation of the thermodynamic properties, such as the temperature dependence of the magnetization and heat capacity, can be obtained from the density of states at $T=0$. The magnetization can be written

$$
M(T)=M(0)-\int_{E_{1}}^{E_{N}} \mathcal{P}(E) f(E) d E,
$$

where $\mathcal{P}(E)$ is the density of states and $f(E)$ is the Bose

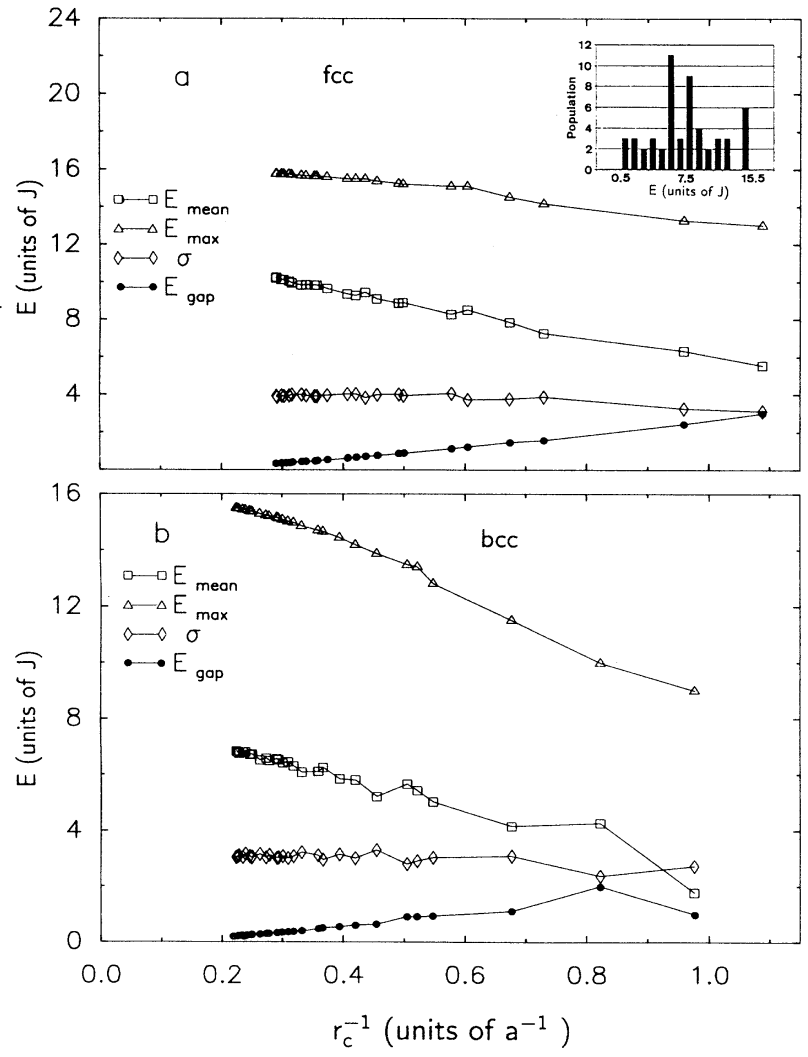

FIG. 6. Characterization of the density of states for fcc (a) and bcc (b) -structured clusters. $\sigma$ is the standard deviation of the density of states. The inset is the density of states for the 55-spin fcc cluster. 
factor. This approach requires an analytical expression for $\mathcal{P}(E)$. In a first approximation a simple analytic form of $\mathcal{P}(E)$ can be chosen, which fulfills the criteria that it has the mean value and standard deviation of the calculated density of states. The success of such an approach depends on how accurately the full density of states is represented by its first two moments $\left(E_{\text {mean }}, \sigma\right)$. (When addressing the thermodynamic properties in the following we use the exact states.)

From Fig. 6 it is immediately clear that thermodynamic properties, such as the temperature dependence of the magnetization and the critical temperature of the clusters, must be different from the bulk properties. The overall softening of the eigenvalues in the clusters will at elevated temperatures (where $\Delta E$ is unimportant) lead to a faster decay of the magnetization with temperature in the clusters than in the bulk. Consequently, the critical temperature for the clusters would be expected to be lower than for the bulk. [This is the case already in a simple mean-field picture, as the Curie temperature here is proportional to the effective coordination $\left(=E_{\text {mean }} / J\right)$.] Further discussion of these considerations is given in the following section, where we calculate the temperature dependence of the magnetization, the critical temperature, and the temperature dependence of the heat capacity for clusters of various structures and sizes.

\section{THERMODYNAMIC PROPERTIES}

\section{A. Magnetization profile}

To calculate the temperature dependence of the magnetization Eqs. (2) and (8) must be solved selfconsistently at various temperatures. This is done as follows. Equation (2) is first diagonalized for an assumed uniform magnetization profile $M_{i}=S$. From the solution $\left(\psi_{i}^{p}\right)$ a better approximation to the magnetization profile at a given temperature is calculated from Eq. (8). The diagonalization of Eq. (2) is then repeated with the new $M_{i}$ profile. By solving Eqs. (2) and (8) iteratively in this manner better and better approximations to the magnetization profile are obtained. The procedure is repeated until the mean magnetization $\left(M_{\text {mean }}\right)$ converges. The procedure is then reinitiated, but this time starting from a uniform magnetization profile with $M_{i}$ smaller than the just obtained $M_{\text {mean }}$ value at convergence. If the same magnetization profile is found, regardless of whether the starting guess is smaller or larger than the value at convergence, the solution is accepted. The temperature dependence of the mean magnetization calculated in this manner for three fcc clusters consisting of 19, 55, and 201 spins is illustrated in Fig. 7. The thin solid curves are extrapolations of the calculated low-temperature behavior to the effective critical temperature of the clusters. The calculation of the effective critical temperatures will be discussed in a later section.

The magnetization of the surface layer decays faster, and the magnetization of the central spin decays slower with temperature than does the average magnetization of the cluster. Hence, a nonuniform magnetization profile evolves (from the uniform ground state) with increasing

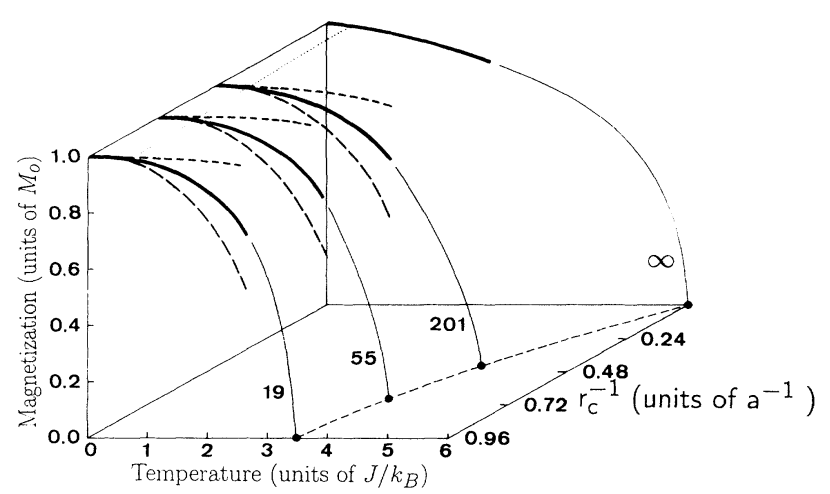

FIG. 7. Mean magnetization (thick solid line), the magnetization at the center (dashed line), and the magnetization of the surface layer (long-dashed line) as a function of temperature for 19, 55, and 201 fcc clusters. The bulk behavior is also sketched $\left(r_{c}^{-1}=0\right)$. Extrapolations to the calculated effective transition temperature $T_{c}$ are shown with thin solid curves, and the trend of the variation of $T_{c}$ with cluster size is illustrated in the $M=0$ plane (thin dashed line). The thin dotted curve is the $M_{\text {mean }}=0.99 M_{0}$ contour.

temperature. The existence of a magnetization profile across the cluster is a consequence of the reduced number of nearest neighbors at the surface of the clusters. At temperatures corresponding to $25 \%$ of the mean-field Curie temperature $\left(T_{C}^{\mathrm{MF}}\right)$ the magnetization varies about $20 \%$ across the cluster. By comparing with the bulk behavior sketched in the back of the figure it is clear that the magnetization of the central spin in the clusters decays even slower with temperature than the bulk magnetization. As spin deviations are energetically cheaper at the surface of the cluster than in the fully coordinated central part, the spin deviations are transferred to the cluster surface.

The effects of finite size is also seen on the temperature dependence of the mean magnetization. At very low temperatures the mean magnetization in the clusters decays slower with temperature than does the magnetization in the bulk. This can be seen from the $M_{\text {mean }}=0.99$ contour in Fig. 7. It is a consequence of the energy gap in the spin-wave spectrum. However, at elevated temperatures the influence of the self-consistent softening of the eigenstates becomes dominating, and the mean magnetization of the clusters decays faster with temperature than the bulk magnetization. These effects become less important with increasing cluster size, and the mean magnetization curve of the clusters approach that of the bulk (cf. Fig. 7$).^{32}$

\section{B. Temperature dependence of the mean magnetization}

In the $T \rightarrow 0$ limit the temperature dependence of the magnetization is exponential,

$$
\lim _{T \rightarrow 0} M_{\text {mean }} \propto S\left[1-\exp \left(\Delta E / k_{B} T\right)\right],
$$

due to the energy gap between the ground state and the 
first excited state. This is a good description as long as the temperature is so low that only the first (few) eigenstates are populated. In Ref. 24 it was shown that the temperature dependence of the mean magnetization of bcc clusters in a larger temperature interval (0-0.25 $T_{C}^{\mathrm{MF}}$ ) was well described with a power law

$$
M_{\text {mean }}=S\left(1-B T^{\alpha}\right) .
$$

We find that this also holds in the case of fcc and disordered clusters. It is by no means obvious that the finitesize effects discussed above should lead to a power law for the temperature dependence of the mean magnetization, and a rigorous theoretical justification of the functional form cannot be given. However, the expression has the right form in the limit of an infinite system, and the two finite-size effects discussed above, namely, the energy gap and the lowering of the mean number of nearest neighbors, result in deviations from the bulk behavior that can be effectively described through larger than bulk values of $\alpha$ and $B$ in Eq. (16). The prime justification of the above approach is that it results in very good fits of the calculated curves and hence allows the finite-size effects to be expressed in a simple and effective way through the parameters $\alpha$ and $B$. Furthermore, it is in accordance with experimental praxis in the field. Only for the smallest clusters appreciable deviations between the power-law fits and the calculated behavior is seen at low temperatures. This is a manifestation of the low-temperature exponential behavior.

Results from fitting the temperature dependence of the mean magnetization of fcc and bcc clusters of various sizes with a power law [Eq. (16)] are shown in Fig. 8, where the exponent $\alpha$ [Fig. 8(a)] and the prefactor $B$ [Fig. 8(b)] (Ref. 33) are plotted as a function of reciprocal size $\left(1 / r_{c}\right)$. The quality of the fits is illustrated in Fig. 9. The exponent $\alpha$ is generally larger than the bulk value $(3 / 2)$ and decreases with increasing cluster size regardless of structure. The observed size trend extrapolates nicely to the known bulk value for $\alpha$. This holds for both bcc- and fcc-structured clusters and allows a prediction of the effective power law for larger clusters than those studied here. The scatter of the points around the overall size trend is due to variations in the configuration of the outermost shells in the cluster $\left(z_{\text {eff }}\right)$. The difference in the slopes of the $\alpha$ versus $r_{c}^{-1}$ lines for the fcc- and bcc-structured clusters is due to the different density of the two structures. A bcc cluster is $2^{1 / 3}$ times larger than a fcc-structured cluster with the same number of spins. If the bcc results are transformed according to $r_{\mathrm{bcc}}^{\prime}=2^{-1 / 3} r_{\mathrm{bcc}}$, such that clusters with the same number of spins have the same abscissa regardless of structure, the two $\alpha$ versus $r_{c}^{-1}$ curves coincide. The exponent of the power-law fit, $\alpha$, is thus size dependent but structure independent. This is important, and we expect it to hold also for other structures (of lower symmetry) which might be present in real clusters.

The $B$ parameter varies in a less systematic way with size than $\alpha$. For the bcc-structured clusters $B$ is generally somewhat larger than the bulk value and for the fcc clusters somewhat lower than the bulk value. The $B$
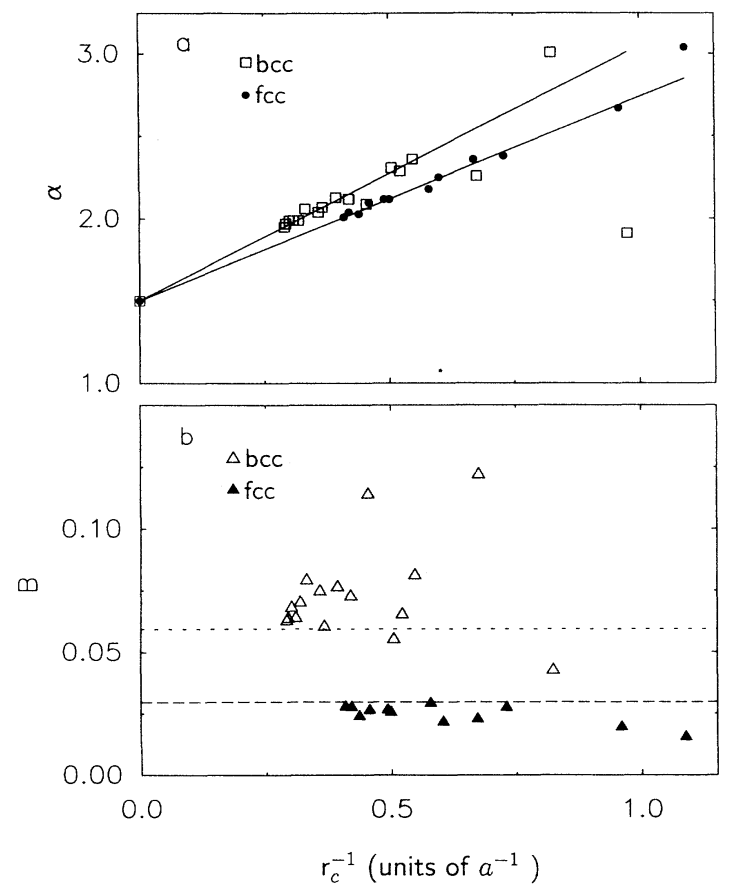

FIG. 8. (a) Effective exponent $\alpha$ of the power-law fits [Eq. (16)] of the temperature dependence of the mean magnetization for fcc $(\bullet)$ and bcc $(\square)$-structured clusters. The straight lines represent simple extrapolations of the observed size trend to the bulk value of $\alpha$. (b) The prefactor $B$ of the power-law fits of the magnetization [fcc (solid triangle), bcc (open triangle)]. The horizontal lines are the $B$ values for the infinite bcc (dashed line) and fcc (long-dashed line) lattices.

value depends on the effective coordination number $z_{\text {eff }}$ in the cluster. As discussed in Sec. III B, the effective coordination number depends critically on the structure of the outermost shells in the cluster (the cluster closure) as well as on the cluster size. As illustrated in Fig. 5, $z_{\text {eff }}$ is not a smoothly varying function of the cluster size. This is the reason for the scatter of the fitted $B$ values. By comparing Figs. 8 and 5 a correlation between the fitted $B$ values and the mean number of neighbors is seen.

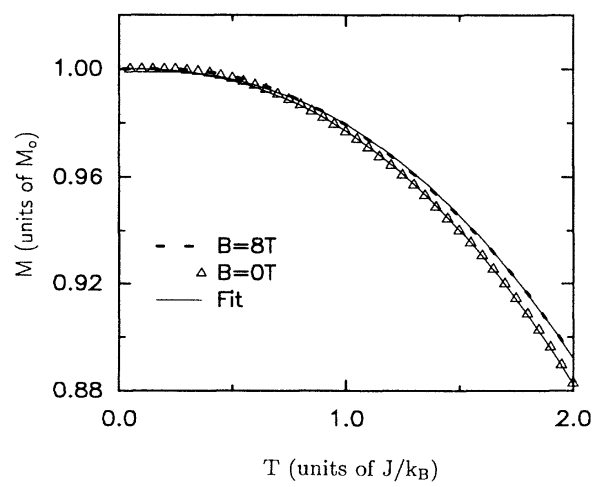

FIG. 9. Temperature dependence of the mean magnetization of a 55-spin fcc cluster in zero field and in a magnetic field of $8 \mathrm{~T}$. The solid curves are best-fit curves using a power law. 
Large $B$ values correspond to "open" clusters with $z_{\text {eff }}$ parameters that lie below the overall size trend. The size dependence of the $B$ parameter can thus be understood when the detailed structure of the outermost shells in the clusters is taken into account. As the importance of the surface of the clusters decreases with increasing size so does the scatter of the $B$ values. It is thus a fair approximation (better than $10 \%$, cf. Fig. 5) to use the bulk $B$ values for cluster sizes beyond those where a full calculation has been done (249 fcc and 339 bcc). For other structures we expect the $B$ values to lie between the limits set by the bcc and fcc structures.

\section{Effects of an external magnetic field or anisotropy}

In the following we consider the influence of a magnetic field and magnetic anisotropy on the magnetic properties of the clusters. (This is of relevance because real clusters presumably possess some inherent magnetic anisotropy and because when measuring the temperature dependence of the magnetization of nanoscale particles a large magnetic field must be applied to quench the superparamagnetic relaxation. ${ }^{6,7}$ ) If the clusters are placed in a magnetic field $(H)$, the equation of motion of $S_{i}^{+}$is modified, as $H$ adds to the molecular field. An extra term $g \mu_{B} H$ is added in the diagonal terms of Eq. (2). ( $g$ is the $g$ factor and $\mu_{B}$ is the Bohr magneton.) This shifts the eigenvalues to higher energies and, hence, results in a slower decay of the cluster magnetization with increasing temperature than experienced without an applied magnetic field. However, the magnetic field has to be very large before the effect becomes significant. This can be realized by the following order-of-magnitude argumentation. The diagonal terms of Eq. (2) are on the order of $M_{\text {mean }} z_{\text {eff }} J$ and cannot be smaller than $M_{\text {surf }} J$. Using values for $\alpha-\mathrm{Fe}\left(J \approx 11.9 \mathrm{meV}\right.$ and $S g \mu_{B} \approx 0.128$ $\mathrm{meV} / \mathrm{T}$ ) we note that the exchange interaction between two neighboring spins in $\alpha$-Fe is about 100 times stronger than the interaction with an applied field of $1 \mathrm{~T}$. The energy gap introduced by the finite size of the clusters considered here was in Sec. III B shown to be $0.2-1.0 \mathrm{~J}$. The contribution of an external field to the energy gap is $g \mu_{B} H$, which is small in comparison with the finitesize effect (taking the values for $\alpha$-Fe to be typical). The effect of an external field is felt most strongly by the outermost spins of the clusters, and it becomes increasingly important with increasing temperature.

By the above order-of-magnitude arguments it is clear that the conclusions drawn about the temperature dependence of the mean magnetization in the previous section are unaltered by the presence of even quite large magnetic fields. As an example of the influence of a magnetic field, the temperature dependence of the mean magnetization of a 55-spin fcc cluster in zero field and in a magnetic field of $8 \mathrm{~T}$ is plotted in Fig. 9. The solid curves are the power-law fits [Eq. (16)]. The exponent of the power-law fit of the mean magnetization increases by only $2 \%$ by applying a field of $8 \mathrm{~T}$. The prefactor decreases about $10 \%$ ( $J$ and $g \mu_{B}$ values of $\alpha \mathrm{Fe}$ were used). Generally, as long as $H \ll J S /\left(g \mu_{B}\right)$ the influence of the magnetic field can be neglected.
The influence of magnetic dipole interactions (shape anisotropy) or crystalline anisotropy in the clusters can in a first approximation be expressed in terms of the effective field they give rise to in the cluster. Experimentally, the magnetic anisotropy is often expressed in terms of a uniaxial anisotropy constant $K$, regardless of the origin of the anisotropy. $K$ generally increases with decreasing particle size. In a study of 6 -nm $\alpha$-Fe particles a value $K \approx 1 \times 10^{5} \mathrm{~J} / \mathrm{m}^{3}$ was found, ${ }^{34}$ and for 2 -nm $\alpha$-iron particles $K \approx 2 \times 10^{6} \mathrm{~J} / \mathrm{m}^{3}$ has been reported. ${ }^{35}$ These anisotropies correspond to effective fields of 0.05 and 1.1 $\mathrm{T}$, respectively. Anisotropy energies of this order of magnitude are thus negligible. As long as $K \ll J S^{2} / a^{3}$ it can be neglected when considering the temperature dependence of the cluster magnetization and the energy gap in the spin-wave spectrum.

\section{Effects of varying the cluster structure}

For both fcc- and bcc-structured model clusters the finite size of the clusters lead to a temperature dependence of the mean magnetization that is well described with a power law [Eq. (16)] with a size-dependent exponent $\alpha$ larger than the bulk value. Since little is known about the structure of real clusters, it is of interest to examine to what extent the observed effects depend on the cluster structure. Therefore, we have performed a number of calculations for clusters that do not posess a perfect fcc or bcc structure. We have, as in Sec. III A, modified the structure in two ways: by moving the spins randomly relative to the underlying lattice by up to $10 \%$ of the nearest-neighbor distance and by introducing holes in the clusters by removing (in a random way) up to $20 \%$ of the spins. When the clusters are not perfectly ordered there exists no unique nearest-neighbor distance. In this case spins that are closer than the nearest-neighbor distance of the underlying lattice plus 2 times the maximum displacement are taken to be nearest neighbors. The exchange energy constant is for simplicity assumed to be either proportional or inversely proportional to the spin separation $\left[J^{\prime}\left(r_{0}\right)= \pm J\left(r_{0}\right) / r_{0}\right]$, resulting in variations of up to $\pm 20 \%$ in $J_{i j}=J\left(r_{0}\right)+\Delta r J^{\prime}\left(r_{0}\right)$.

Disordering a 225-spin cluster by random displacements of the spins relative to an underlying fcc lattice has no effect on the temperature dependence of the mean magnetization. This dependence is still described well with a power law, and the fitting parameters $\alpha$ and $B$ are as for the undisturbed cluster. This is found no matter whether the exchange energy constant is proportional or inversely proportional to the spin separation, i.e., regardless of the sign of $J^{\prime}\left(r_{0}\right)$. The parameters $\alpha$ and $B$ only depend on the eigenvalue spectrum. The variations of the eigenvalues introduced by the disturbances in the spin positions depend on the product $\Delta r J^{\prime}\left(r_{0}\right)$. Hence the power-law description will be valid also for more realistic values of $J^{\prime}\left(r_{0}\right)\left[\left|J^{\prime}\left(r_{0}\right)\right| \gg J\left(r_{0}\right) / r_{0}\right]$.

Introducing holes at up to $20 \%$ of the spin sites in the clusters does not change the functional form of the temperature dependence of the mean magnetization. However, the factor $B$ is found to change. The effects of picking out spins $(5,10, \ldots, 40)$ of a disordered cluster 
originally being a 225-spin fcc cluster are summarized in Fig. 10, where $\alpha$ and $B$ of power-law fits to the mean magnetization are plotted versus $z_{\text {eff }}$ of the clusters. The horizontal lines indicate the $B$ values of the undisturbed cluster (long-dashed line) and of a 181-spin bcc cluster (short-dashed line). The $\alpha$ values of these clusters are also shown for comparison. Picking out spins lowers the coordination number of the cluster, and in accordance with what was found in Sec. IV B for the bcc clusters, this results in an increase of $B$. Reducing the coordination number, from the value typical for the fcc cluster to a value closer to that typical of a bcc cluster, results in an increase in $B$ from the fcc value (long-dashed line) to the bcc value (short-dashed line). The exponent $\alpha$ varies only little with the introduction of holes. For a cluster containing 40 holes $\alpha$ is only about $5 \%$ lower than for the undisturbed cluster. The parameters (especially $B$ ) of the power-law fit are dependent on which spins are taken out of the clusters. This is illustrated in Fig. 10, where the effects of picking out 40 spins in different ways are illustrated. The spread of the pluses illustrates the characteristic spread on each of the points in Fig. 10.

In conclusion, the temperature dependence of the mean magnetization can be well described with a power law regardless of the cluster structure. The exponent $\alpha$ depends primarily on $N$ and only very vaguely on structure. The factor $B$, on the other hand, depends on the effective coordination of the cluster in a simple manner. The fcc and bcc lattices represent the extremes with regard to coordination number, and the $B$ parameters of other structures are therefore expected to lie between the extremes set by these two structures, as was also found for the disordered clusters with holes.

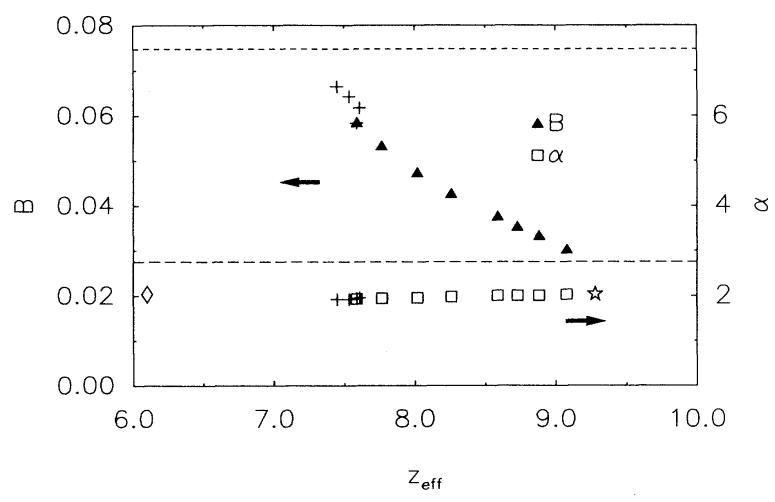

FIG. 10. Influence on the parameters of the power-law fits, $\alpha(\square)$ and $B$ (solid triangle), of introducing holes in a 225-spin "fcc" cluster (see text). The fitting parameters are plotted as a function of the effective coordination number $z_{\text {eff }}$. The scale for $\alpha$ is given on the right and the scale for $B$ on the left. The star is the $\alpha$ value of the undisturbed fcc cluster, and the diamond the $\alpha$ value of a 181-spin bcc-structured cluster. The two horizontal lines indicate the values of the $B$ parameters for the 225-spin fcc cluster (long-dashed line) and for the 181-spin bcc cluster (short-dashed line). The pluses are the fitting parameters for a cluster where 40 holes have been introduced at different random sites.

\section{E. Effective critical temperature}

The rapid decrease of the magnetization and the overall softening of the eigenstates at high temperature influence the effective critical temperature $\left(T_{c}\right)$ of the clusters. In the bulk $(N \rightarrow \infty)$ the correlation theory for ideal Heisenberg magnets ${ }^{36}$ and the spherical approximation predict the correct $T_{c}$ within a few percent of the best available high-temperature expansion estimates:

$$
\begin{aligned}
\frac{T_{C}^{\mathrm{MF}}}{T} & =\frac{1}{N} \sum_{\mathbf{q}} \frac{J_{0}}{\left(1 / \chi^{o}-J_{\mathbf{q}}\right)} \\
& =\frac{1}{N} \sum_{\mathbf{q}} \frac{1}{\left(1 / \chi+\hbar \omega_{\mathbf{q}} / J_{0} S\right)} .
\end{aligned}
$$

$T_{C}^{\mathrm{MF}}$ is the mean-field Curie temperature $\left(T_{C}^{\mathrm{MF}}=\right.$ $\left.J_{0} S(S+1) / 3 k_{B}\right), \chi_{0}$ is the noninteracting spin susceptibility, and $\chi$ is the uniform spin susceptibility (see also the Appendix). The uniform spin susceptibility diverges at the critical temperature, which is thus calculated from Eq. (17) by setting $1 / \chi=0$. This approach can, as shown in the Appendix, be generalized to the case of clusters, yielding

$$
\frac{T_{C}^{\mathrm{MF}}}{T}=\frac{1}{N-1} \sum_{p=1}^{N-1} \frac{1}{1 / \chi+E_{p} / J_{0} S},
$$

where $E_{p}$ are the eigenvalues of Eq. (2), and $\chi$ is the cluster analog of the uniform spin susceptibility in the infinite system. The temperature dependence of $\chi$ can be obtained from Eq. (18). By analogy with the procedure for the infinite system, the temperature calculated from Eq. (18) for $1 / \chi=0$ is identified as an effective critical temperature for the clusters. It is thus possible to estimate the effective $T_{c}$ of the clusters from a knowledge of the spin-wave spectrum $\left(E_{p}\right)$ at $T=0$, similarly to the bulk case.

In Fig. 11 the temperature dependence of the inverse susceptibility $(1 / \chi)$ for three different sized $(55,225$, and $381)$ fcc clusters is illustrated. Also the temperature dependence of the inverse mean-field susceptibility of the 225-spin cluster and the bulk (the Curie-Weiss law) are

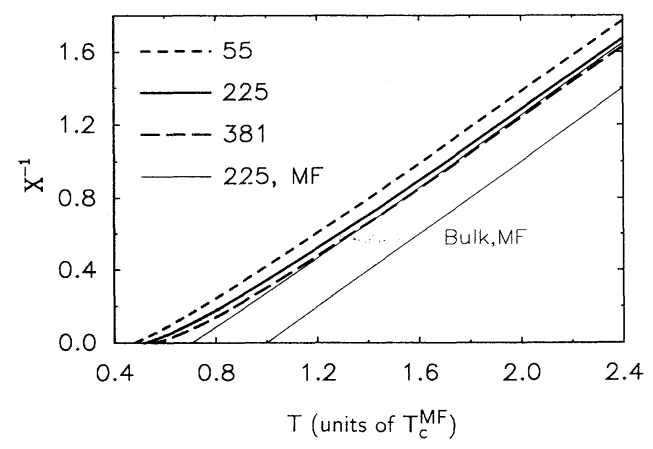

FIG. 11. Inverse susceptibility $\chi^{-1}$ as a function of temperature for fcc clusters of various sizes calculated from Eq. (18). The mean-field behavior is also sketched for the infinite system and for the 225-spin cluster. 
illustrated. The lowering of the coordination number in the clusters leads to a shift to lower temperatures of the inverse susceptibility curves. This effect is included in a mean-field picture, where the critical temperature is proportional to the coordination number $\left(z_{\text {eff }}\right)$ (compare the bulk mean-field curve and the 225-cluster mean-field curve). The correlation effects leads to a curvature of the inverse susceptibility curves, as can be seen by comparing the mean-field curve and the curve calculated from Eq. (18) for the 225-spin cluster in Fig. 11. The horizontal shift of the curves to lower temperatures decreases, as expected, with increasing cluster size. The curvature of the curves at low temperature gets more and more pronounced with increasing cluster size-the transition gradually approaches a real phase transition with critical fluctuations in the thermodynamic limit. Note that a determination of the magnetic moment of the system from a straight-line fit to measured inverse susceptibility data is troublesome due to the curvature of the inverse susceptibility curves.

The intersection with the $T$ axis is the critical temperature. The finite-size reduction of the critical temperature is thus much stronger than predicted by a mean-
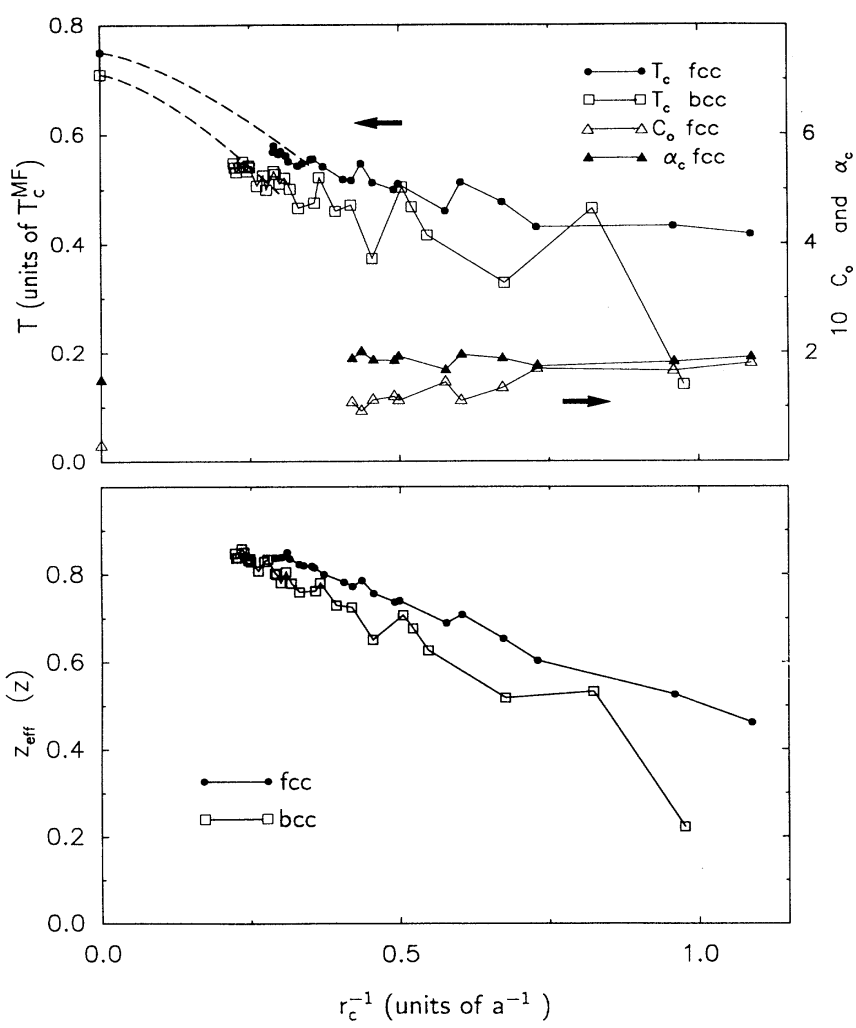

FIG. 12. (a) Size dependence of $T_{c}$, calculated from Eq. (18), for fcc $(\bullet)$ and bcc $(\square)$ clusters. The dashed curves are extrapolations to the bulk high-temperature expansion value of $T_{c}$ using a finite-size scaling law (see text). The parameters of a power-law fit, $\alpha_{c}$ and $C_{0}$, of the temperature dependence of the spin-wave heat capacity are shown as solid and open triangles, respectively. (b) The effective coordination number $z_{\text {eff }}$ (mean number of nearest neighbors) for all the considered bcc $(\square)$ and fcc $(\bullet)$ clusters. field-type argument. In Fig. 12, $T_{c}$ calculated from Eq. (18) is plotted versus $1 / r_{c}$ for fcc and bcc clusters. A size-dependent decrease of $T_{c}$ by up to about $50 \%$ of the bulk high-temperature expansion value of $T_{c}$ is seen for the smallest clusters. The dashed lines in Fig. 12 are interpolations between $T_{c}$ of the bulk and of the largest clusters, using the finite-size scaling law $\left[T_{c}\left(r_{c}\right)-T_{c}(\infty)\right] / T_{c}(\infty)=-\left(r_{c} / r_{0}\right)^{-1 / \nu}$ (Ref. 37) with $2 \nu=1.4, r_{0}$ (bcc) $=0.725 a$, and $r_{0}($ fcc $)=0.57 a$.

The scatter of the points around the overall size trend in Fig. 12 is the already discussed surface effect. To illustrate this the variation of $z_{\text {eff }}$ with size is reproduced for both fcc- and bcc-structured clusters in the lower half of Fig. 12. The correlation between the size dependence of $T_{c}$ and $z_{\text {eff }}$ is quite clear.

The direct dependence of $T_{c}$ on the mean coordination number is also seen when considering disordered clusters. In Fig. 13, $T_{c}$ calculated from Eq. (18) is shown as a function of $z_{\text {eff }}$. The mean coordination number was varied by removing an increasing number of spins from a disordered "fcc" cluster originally containing 225 spins (cf. Sec. IV D). The critical temperature varies linearly with $z_{\text {eff }}$, as expected from a mean-field-type argument. The slope of the line is approximately $1 / z=1 / 12$, as expected from the mean-field picture.

In Fig. 7 the critical temperatures calculated from Eq. (18) were plotted in the $M=0$ plane. The calculated critical temperatures are consistent with the behavior of the mean magnetization at lower temperatures, since $M_{\text {mean }}(T)$ extrapolates quite nicely to the calculated $T_{c}$ 's.

Monte Carlo studies have shown that a phase transition in the usual sense does not exist in finite-size Heisenberg systems. ${ }^{20}$ Because of the finite size of the system, it never becomes truly paramagnetic, as there will always be a nonzero magnetization proportional to $N^{-1 / 2}$. One can define an effective critical temperature as the temperature at which the derivative of the magnetization, with respect to temperature, adopts its maximum numerical value. In the Monte Carlo studies this temperature is found to decrease with decreasing size of the system, ${ }^{20}$ in qualitative agreement with our findings.

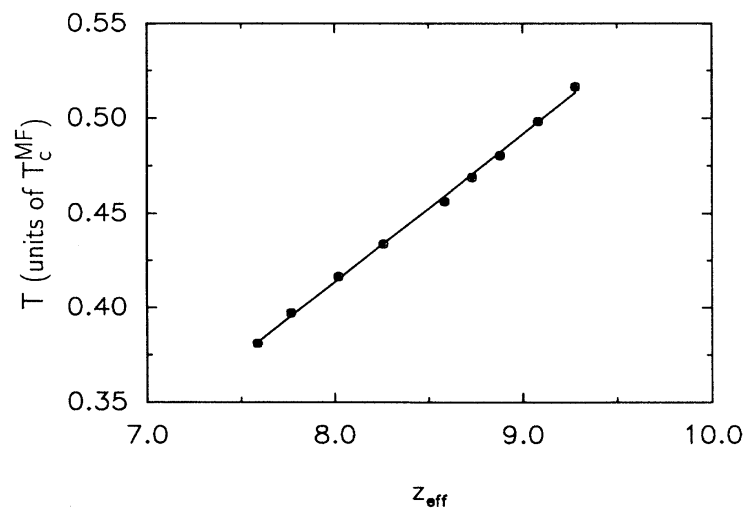

FIG. 13. Dependence of the effective transition temperature $T_{c}$ on the mean number of nearest neighbors in the disordered clusters with holes (see text). 
The coefficients of a high-temperature expansion of the susceptibility have been calculated for a finite simple cubic system. ${ }^{38}$ This study also predicts a size-dependent reduction of $T_{c}$ relative to the Curie temperature of the bulk.

\section{F. Spin-wave contribution to the heat capacity}

The spin-wave contribution to the heat capacity $(C=$ $d U / d T)$ of the clusters can be calculated from the internal energy per atom $U$ :

$$
U=\frac{1}{N} \sum_{p=1}^{N-1} \frac{E_{p}}{\exp \left(E_{p} / k_{B} T\right)-1} .
$$

A self-consistent calculation of $U$ as a function of temperature can be performed simultaneously with the calculation of the temperature dependence of the magnetization. The finite size of the clusters causes a modification of the bulk $T^{3 / 2}$ law for the spin-wave contribution to the heat capacity. The influence of the energy gap is seen in the low-temperature limit. Here the heat capacity of the clusters increases more slowly with temperature than in the bulk. At elevated temperatures the softening of the eigenstates is seen through a much faster increase of $C$ with temperature for the clusters than for the bulk.

The deviation from bulk behavior is much more pronounced for the heat capacity than for the magnetization. The temperature dependence of $M_{\text {mean }}$ of the clusters depends on the spin-wave spectrum $\left(E_{p}\right)$ through the statistical weight factors. The internal energy depends directly on the eigenvalues $E_{p}$ in addition to the indirect dependence through the weight factors. It is therefore not surprising that the finite-size effects are seen even more strongly on a quantity such as the heat capacity than on the magnetization.

Following the data analysis of the temperature dependence of the mean magnetization we have tried to fit the temperature dependence of the heat capacity with a power law $\left(C=C_{0} T^{\alpha_{c}}\right)$. Results of the fitting are summarized in Fig. 12 for fcc-structured clusters (right scale). Whereas this approach in the case of the magnetization was fully justified by the quality of the fits, it is more questionable in the case of $C$ since the fits are not nearly as good. However, it is maintained to give a simple quantitative measure of the finite-size effects. Both $C_{0}$ and $\alpha_{c}$ for the clusters are found to be larger than the bulk values $\left[\alpha_{c}=1.5, C_{0}=0.0283\right.$ (Ref. 26)] (see Fig. 2 for $r_{c}^{-1}=0$ ). As can be seen from Fig. 12, the scatter of the coefficients $\alpha_{\mathrm{c}}$ and $C_{0}$, expressing the temperature dependence of $C$, is strongly correlated with $z_{\text {eff }}$. The same was observed for $T_{c}$. It is a consequence of the direct dependence on the eigenvalues of both $T_{c}$ and $C$.

\section{COMPARISON TO EXPERIMENTAL DATA}

\section{A. Critical temperature}

A recent study of Gd clusters seems to indicate an enhancement of $T_{c}$ as compared with bulk Gd. ${ }^{39}$ For rela- tively large ferrimagnetic $\mathrm{MnFe}_{2} \mathrm{O}_{4}$ particles larger than bulk Curie temperatures have also been reported. ${ }^{40}$ It has been questioned whether the $T_{c}$ enhancement in the $\mathrm{MnFe}_{2} \mathrm{O}_{4}$ study is an effect of the finite size or an effect of a varying cation distribution. ${ }^{41}$ The experimental findings are in conspicuous contradiction to our findings for the thermodynamic properties of Heisenberg model clusters.

It is important to realize what effects have been neglected in the present study. We have assumed the exchange energy constant to be equal to the bulk value and to be the same among all neighboring spins. Furthermore, we have assumed the magnetic moment to be the same at all sites in the cluster. Electronic structure calculations (at $T=0$ ) have shown that at least the last assumption is doubtful. It has been shown that the magnetic moment of the atoms on the surface is larger than for the atoms in the center of the cluster. ${ }^{11}$ Such an effect will to some extent counterbalance the effect of the lowering of the coordination number at the surface, which was found to be important for the thermodynamic properties of the model clusters in this study. A general increase in the magnetic moment as reported for $\mathrm{Co},{ }^{6}$ $\mathrm{Fe},{ }^{7}$ and Gd clusters ${ }^{39}$ will result (if it was the only effect of finite size) in an increase of $T_{c}$ compared with bulk values. The experimental results indicating an enhancement of $T_{c}$ compared with bulk values imply that electronic changes must be of considerable importance - the lowering of $T_{c}$ predicted from the treatment of the statistical properties must be counterbalanced either through enhanced moments or increased strength of the exchange interaction. If these electronic effects were known quantitatively, the presented method could be used to calculate the statistical effects, which should be similar to those discussed above.

\section{B. Temperature dependence of the magnetization}

Although in the last few years there has been a great deal of interest in ultrafine ferromagnetic particles, only a few studies have been dedicated to a determination of the temperature dependence of the magnetization. A comprehensive list of references on studies of ultrafine iron particles and clusters can be found in Ref. 24 . It does, however, seem well established experimentally that the magnetization is not homogeneous in ultrafine particles. ${ }^{4,42,43}$ The magnetization of the spins near the surface is found to decay faster with temperature than in the bulk. This is in accordance with the predictions of the spin-wave theory for a cluster presented in this paper and with the results of Monte Carlo studies of Heisenberg clusters. $^{23}$

Linderoth et al. have studied the temperature dependence of the magnetization of 3.1-nm amorphous ironcarbon particles ${ }^{25}$ prepared by thermal decomposition of $\mathrm{Fe}(\mathrm{CO})_{5}$. They measured the magnetization of the particles in an applied field of $5 \mathrm{~T}$, using a superconducting quantum interference device (SQUID) magnetometer. The particle-size distribution characteristic of the preparation technique is very narrow. The particles were demonstrated to be magnetically noninteracting. ${ }^{25}$ Their 
results on the temperature dependence of the magnetization are reproduced in Fig. 14. The magnetization does not follow the bulk $T^{3 / 2}$ law. However, the magnetization curve can be fitted well ${ }^{25}$ with a power law [Eq. (16)] with an exponent equal to 1.86 and and a prefactor of $6.5 \times 10^{-6} \mathrm{~K}^{-1.86}$. (The fit is included in Fig. 14 as a solid curve.) The exponent of the effective power law of a model cluster with the same size as the investigated particles should be approximately 1.76 . Considering the simplicity of the model cluster the quantitative agreement between $\alpha$ of the 3.1-nm amorphous iron-carbon particles and that of a Heisenberg model cluster with the same size is good. It therefore seems reasonable to ascribe the observed experimental behavior to the finitesize effects in the spin-wave spectrum.

The prefactor $B$ depends sensitively on the mean coordination number of the spins in the cluster. Assuming that the carbon can be considered as holes in the lattice the model calculations predict larger than bulk values for $B$. This is indeed observed in the experimental studies of amorphous iron-carbon particles, where a $B$ value twice as large as that of $\alpha$ iron is found. ${ }^{25}$

The situation is, however, not quite as settled as the above comparison might suggest. In Ref. 3 the bulk $T^{3 / 2}$ law (with larger than bulk values of the prefactor $B$ ) was found to account well for the behavior of 2-nm $\alpha$-iron particles in a $\mathrm{SiO}_{2}$ matrix. In Ref. 42 a linear temperature dependence of the magnetization of 6 -nm $\alpha$-iron particles in paraffin was reported on the basis of a Mössbauer spectroscopy study. There are a number of factors that complicates experimental studies of the implications of finite size on the magnetic properties. (1) A large fraction of the atoms in nanometer-sized particles is situated at the surface or close to the surface, which makes the properties of nanometer-sized particles sensitive to the influence of the supporting material. It is difficult to separate the influence of the support from the intrinsic properties. (2) Direct magnetic interactions between the particles in the sample can be of importance. (3) The influence of mag-

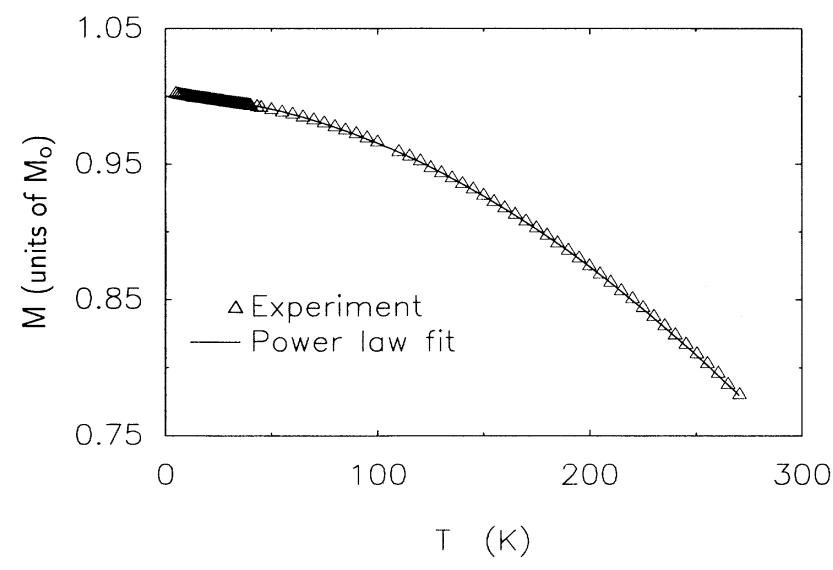

FIG. 14. Data of Linderoth et. al. (Ref. 25) for the temperature dependence of the magnetization of 3.1-nm amorphous iron-carbon particles $(\triangle)$. The solid curve is a best-fit curve with a power law $\left(M=1-B T^{\alpha}\right)$. netic relaxation phenomena, such as collective magnetic excitations, ${ }^{44}$ has to be considered. (4) Due account of the particle-size distribution must be taken. These effects will vary in importance from system to system. Further experimental work is needed to clarify the detailed implications of finite size on the thermodynamic properties of clusters and nanoparticles.

As discussed previously the finite size might also affect electronic properties, resulting in exchange energy constants that vary with position and in site-dependent magnetic moments different from the bulk value. A more advanced model of a metal cluster must include a treatment of the electronic properties in addition to the treatment of the statistical properties.

\section{CONCLUSIONS}

By a calculation of the spin-wave spectrum of Heisenberg model clusters of different size and structure we have addressed the thermodynamic properties of magnetic clusters and ultrafine particles. In particular, we have elucidated the effects of the finite system size in the thermodynamic properties.

The spin-wave spectrum is found to be discrete in energy and wave-vector broadened, resulting in a neutronscattering cross section very different from that of the bulk. The finite size results in a number of energy gaps in the spectrum. The energy gap between the ground state and the first excited state is of particular importance $(\sim 30 \mathrm{~K}$ for an iron cluster containing 725 spins $)$. A simple picture relating the energy gap to the characteristic wavelength of the first eigenstate is found to account well for the size dependence of the energy gap.

Both the energy gap and the wave-vector broadening in the spin-wave spectrum should be readily observed in a neutron-scattering experiment.

The finite size was found to lead to a nonuniform magnetization profile decreasing toward the surface in the clusters and to a temperature dependence of the mean magnetization different from the Bloch $T^{3 / 2}$ law. We find that the behavior of the mean magnetization is well described by a power law with a size-dependent exponent that is larger than for the bulk. The exponent is insensitive to the structure, but the prefactor depends on the detailed cluster structure.

A substantial size-dependent reduction of the effective critical temperature of the clusters compared to the bulk high-temperature expansion values was found from calculations based on a generalization of the spherical approximation to finite-sized systems. This was found regardless of the cluster structure. (Bulk values for the exchange interaction and saturation magnetization were assumed.)

When considering a real system, finite-size effects in the electronic properties might have to be taken into account in addition to the statistical finite-size effects treated in the present work. Experimental evidence exists, ${ }^{25}$ that the finite-size effects discussed for the Heisenberg clusters do manifest themselves in a real system. 


\section{APPENDIX A: CALCULATION OF THE EFFECTIVE TRANSITION TEMPERATURE}

For three-dimensional spin systems an accurate estimate of the transition temperature $T_{c}$ can be obtained using the correlation theory ${ }^{36}$ and the spherical approximation. ${ }^{45}$ The idea is to calculate the wavevector-dependent susceptibility $\chi_{q}$ and to use the exact sum rule for the constant spin length. The transition temperature is in the thermodynamic limit determined by the condition that $\chi_{q} \rightarrow \infty$ for the ordering vector $\mathbf{q}=\mathbf{Q}$ when $T \rightarrow T_{c}$. For the ferromagnetic case $Q=0$. This condition was used in Ref. 38 in connection with a high-temperature expansion. For a finite cluster the susceptibility cannot diverge, but we expect it to show a maximum $\propto N$ at an effective transition temperature. This transition temperature is then determined by the criterion for finding the largest component of $\chi_{q}$. The caution that the susceptibility cannot tend to infinity, but must be replaced by a value proportional to $N$, was already pointed out by Binder, Rauch, and Wildpaner. ${ }^{20}$

For an infinite Heisenberg spin system with translational symmetry it is easy to show that the equation of motion of $S^{+}$and the equation for the nonlocal susceptibility are diagonalized by the same transformation, the Fourier transformation. The nonlocal susceptibility is

$\chi_{i j}=\frac{\partial M_{i}}{\partial h_{j}}, \quad \partial M=\sum_{i=1}^{N} \partial M_{i}=\sum_{i, j}^{\text {all }} \chi_{i j} \partial h_{j}$,

where $\partial M_{i}$ is the magnetization induced at site $i$ by a small magnetic field $\partial h_{j}$ at site $j ; \partial M$ is the total induced moment. We consider only fields in the $z$ direction. The total field $H_{i}$ acting on a spin includes the molecular field from the neighbor spins. It is convenient first to take the derivative with respect to $H_{i}=\sum_{j} J_{i j} M_{j}+h_{i}$. This gives

$$
\chi_{i j}=\frac{\partial M_{i}}{\partial H_{i}} \frac{\partial H_{i}}{\partial h_{j}}=\chi_{i i}^{0}\left(\sum_{l} J_{i l} \chi_{l j}+\delta_{i j}\right) .
$$

Assuming translational symmetry and that all spins react similarly to the molecular field, we have that $\chi_{i i}^{0}=\chi^{0}$; i.e., it is independent of the site $i$. Then we can Fourier transform Eq. (A2), yielding $\chi_{q}=\chi^{0}\left(J_{q} \chi_{q}+1\right)$. Solving this we can write

$$
\chi_{q}=\frac{1}{1 / \chi^{0}-J_{q}} .
$$

An important constraint is that the length of the spins must be constant: $\mathbf{S}^{2}=S(S+1)$. This gives, for $T>T_{c}$, the sum rule that

$$
\begin{aligned}
1 / N \sum_{i}\left\langle S_{i}^{z} S_{i}^{z}\right\rangle & =S(S+1) / 3 \\
& =1 / N^{2} \sum_{q}\left\langle S_{q}^{z} S_{-q}^{z}\right\rangle \\
& =k_{B} T / N \sum_{q} \chi_{q}
\end{aligned}
$$

where the last equation is obtained from the fluctuationdissipation theorem. In the mean-field theory $\chi^{0}=S(S+$ $1) /\left(3 k_{B} T\right)$ and $T_{C}^{\mathrm{MF}}=J_{0} S(S+1) /\left(3 k_{B}\right)$. Combining
Eqs. (A3) and (A4) we can write

$$
\begin{gathered}
\frac{S(S+1)}{3}=\frac{k_{B} T}{J_{0}} \frac{1}{N} \sum_{q} \frac{1}{1 /\left(\chi^{0} J_{0}\right)-\gamma_{q}}, \\
\frac{T_{C}^{\mathrm{MF}}}{T}=\frac{1}{N} \sum_{q} \frac{1}{R-\gamma_{q}} \\
=\frac{1}{N} \sum_{q} \frac{1}{R-1+\hbar \omega_{q} /\left(J_{0} S\right)} \\
=\frac{1}{N} \sum_{q} \frac{1}{1 / \chi+\hbar \omega_{q} /\left(J_{0} S\right)},
\end{gathered}
$$

where $\chi=1 /(R-1)$ is the uniform spin susceptibility, $\gamma_{q}=J_{q} / J_{0}$, and $\hbar \omega_{q} / J_{0} S$ is the spin-wave energy in units of the molecular field. The parameter $R$ is a better choice than the molecular field value for the inverse noninteracting susceptibility $1 / \chi^{0}$, in units of $J_{0}$. When $R \rightarrow 1$ the $q=0+\operatorname{term}(\chi)$ in the sum diverges (we exclude the term with $q \equiv 0)$. The $T$ which is given by Eq. (A6) for $R=1$ will be defined as the new $T_{c}$. This is the so-called spherical approximation. For $R>1$ it allows a determination of the susceptibility $(\chi)$ for $T>T_{c}$. We shall now see that this can be generalized to the finite cluster case, when instead of summing over the quantum number $q$ we sum over the discrete quantum number $p$. In the last formulation in Eq. (A6) we have expressed the interesting fact that the susceptibility at high temperatures is given in terms of the spin-wave energy $\hbar \omega_{q}$ at $T=0$.

It is useful to consider Eq. (A2) as an $N \times N$ matrix equation

$$
\underline{\chi}=\chi^{0}(\underline{J} \cdot \underline{\chi}+\underline{1}),
$$

where $\chi^{0}$ is a number and $\underline{1}$ is the unit matrix. The Fourier transformation can be considered as a matrix diagonalization by the matrix $\underline{T}$ with the components $T_{l j}=\delta(l j) \exp i \mathbf{q} \cdot \mathbf{r}_{j}$. Consider now the equation of motion for spin waves [Eqs. (2) and (3)] at $T=0$ when $M_{i}=S$ :

$$
\hbar \omega S_{i}^{+}=S \sum_{l} J_{i l}\left(S_{i}^{+}-S_{l}^{+}\right)
$$

or written in matrix form:

$$
\underline{S}^{+}=P\left(\underline{J} \cdot \underline{S}^{+}+\underline{0}\right), \quad P=S /\left(J_{0}-\hbar \omega\right) .
$$

$P$ is a number, and $\underline{0}$ is a zero matrix. It is now clear that the same matrix $T$ which diagonalizes the equation of motion [Eq. (A8)], $\underline{T} \underline{J} \underline{T}^{\dagger}=$ diagonal, also diagonalizes the equation for $\underline{\chi}$ [Eq. (A7)]. This is, of course, true for translational symmetry. However, we have here demonstrated that it holds in general, i.e., also for the numerical diagonalization done for the finite clusters. We can, therefore, generalize Eq. (A6) to

$$
\begin{aligned}
\frac{T_{C}^{\mathrm{MF}}}{T} & =\frac{1}{N-1} \sum_{p=1}^{N-1} \frac{1}{R-1+E_{n} /\left(J_{0} S\right)} \\
& =\frac{1}{N-1} \sum_{p=1}^{N-1} \frac{1}{1 / \chi+E_{n} /\left(J_{0} S\right)},
\end{aligned}
$$


where $E_{n} / J_{0} S$ are the energy eigenvalues in the units of the molecular field and then $T_{C}^{\mathrm{MF}}$ the bulk mean-field transition temperature. We have here excluded the $p=0$ mode, which corresponds to the ground state in which all spins are parallel $\left(E_{0}=0\right)$. The transition temperatures for the clusters calculated from Eq. (A9) are shown in Fig. 12. We note that the extrapolation of the low- temperature behavior of the magnetization (cf. Fig. 7) is in quite good agreement with the $T_{c}$ obtained by using the maximum susceptibility criterion. It is clear from Eq. (A9) that the energy gap $\Delta E=E_{1}-E_{0}$ plays a very significant role in the determination of $T_{c}$. Therefore is is not unexpected that the dependence on the effective coordination number is similar for $\Delta E$ and $T_{c}$.
${ }^{*}$ Present address: Materials Department, Ris $\varnothing$ National Laboratory, DK-4000 Roskilde, Denmark.

${ }^{1}$ W. A. de Heer, P. Milani, and A. Châtelain, Phys. Rev. Lett. 65, 489 (1990).

${ }^{2}$ J. P. Bucher, D. C. Douglass, and L. A. Bloomfield, Phys. Rev. Lett. 66, 3052 (1991).

${ }^{3}$ G. Xiao and C. L. Chien, J. Appl. Phys. 61, 3308 (1987).

${ }^{4}$ F. Bødker, S. Mørup, C. A. Oxborrow, S. Linderoth, M. B. Madsen, and J. Niemantsverdriet, J. Phys. Condens. Matter. 4, 6555 (1992).

${ }^{5}$ C. Djega Mariadassou and J. L. Dormann, in Magnetic Properties of Fine Particles, edited by J. L. Dormann and D. Fiorani (North-Holland, Amsterdam, 1992).

${ }^{6}$ S. N. Khanna and S. Linderoth, Phys. Rev. Lett. 67, 742 (1991).

${ }^{7}$ S. Linderoth and S. N. Khanna, J. Magn. Magn. Mater. 104-107, 1574 (1992).

${ }^{8}$ A. E. Berkowitz, in Magnetic Properties of Fine Particles, edited by J. L. Dormann and D. Fiorani (North-Holland, Amsterdam, 1992).

${ }^{9} \mathrm{~S}$. W. Charles and J. Poppelwell, in Ferromagnetic Materi$a l s$, edited by E. P. Wohlfarth (North-Holland, Amsterdam, 1980), Vol. 2, Chap. 8.

${ }^{10}$ D. M. Cox, D. J. Trevor, R. L. Whetten, E. A. Rohlfing, and A. Kaldor, Phys. Rev. B 32, 7290 (1985).

${ }^{11}$ G. M. Pastor, J. Dorantes-Dávila, and K. H. Bennemann, Phys. Rev. B 40, 7642 (1989).

${ }^{12}$ C. Y. Yang, K. H. Johnson, D.R. Salahub, J. Kaspar, and R. P. Messmer, Phys. Rev. B 24, 5673 (1981).

${ }^{13}$ K. Lee, J. Callaway, and S. Dhar, Phys. Rev. B 30, 1724 (1984).

${ }^{14}$ W. P. Halperin, Rev. Mod. Phys. 58, 533 (1986).

${ }^{15}$ B. I. Dunlap, Phys. Rev. A 41, 5691 (1990).

${ }^{16}$ J.-Y. Yi, D. J. Oh, and J. Bernholc, Phys. Rev. Lett. 67, 1594 (1991).

${ }^{17}$ J. Mansikka-aho, M. Manninen, and E. Hammarén, Z. Phys. D 21, 271 (1991).

${ }^{18}$ H. T. Diep, S. Sawada, and S. Sugano, Phys. Rev. B 39, 9252 (1989).

${ }^{19}$ P. J. Jensen, S. Mukherjee, and K. H. Bennemann, Z. Phys. D 21, 349 (1991).

${ }^{20} \mathrm{~K}$. Binder, H. Rauch, and V. Wildpaner, J. Phys. Chem. Solids 31, 391 (1970).

${ }^{21} \mathrm{~K}$. Binder, Physica 62, 508 (1972).

${ }^{22}$ J. Merikoski, J. Timonen, J. Manninen, and P. Jena, Phys. Rev. Lett. 66, 938 (1991).

${ }^{23}$ V. Wildpaner, Z. Phys. 270, 215 (1974).

${ }^{24}$ P. V. Hendriksen, S. Linderoth, and P.-A. Lindgård, J. Phys. Condens. Matter. 5, 5675 (1993).

${ }^{25}$ S. Linderoth, L. Balcells, A. Labarta, J. Tejada, P. V. Hendriksen, and S. Sethi, J. Magn. Magn. Mater. (to be pub- lished).

${ }^{26}$ D. H. Martin, Magnetism in Solids (MIT Press, Cambridge, MA, 1967).

${ }^{27}$ M. J. Klein and R. S. Smith, Phys. Rev. 81, 378 (1951).

${ }^{28}$ W. Döring, Z. Naturforsch. 16a, 1008 (1961).

${ }^{29}$ For the fcc clusters $r_{0}$ of the smallest cluster $\left(r_{0}=0.92 a\right)$, which contains 13 spins, was used. In the bcc case the radius $r_{0}$ of the second smallest cluster $(N=15)$ was used instead of the value for the smallest cluster $(N=9)$. The 9 -spin cluster is somewhat special: The 8 spins of the first shell are not within nearest-neighbor distance and are thus noninteracting in our model. This makes the 9-spin cluster unrepresentative of the bcc clusters. Furthermore, the lowest-lying eigenstate of the 9-spin bcc cluster does not resemble a standing wave in the [110] direction as does the lowest-lying state in the 15-spin cluster.

${ }^{30}$ J. A. Stratton, Electromagnetic Theory (McGraw-Hill, New York, 1941).

${ }^{31}$ C. Kittel, Introduction to Solid State Physics (Wiley, New York, 1971).

${ }^{32}$ P. V. Hendriksen, S. Linderoth, and P.-A. Lindgård, J. Magn. Magn. Mater. 104-107, 1577 (1992).

${ }^{33}$ The $B$ value for the smallest bcc cluster $\left(N=9, r_{c}^{-1}=\right.$ $0.98)$ is 0.46 . It is out of the scale of Fig. 8.

${ }^{34}$ S. Mørup, B. S. Clausen, and H. Topsøe, J. Phys. (Paris) Colloq. 41, C1-331 (1981).

${ }^{35}$ C. L. Chien, in Science and Technology of Nanostructured Magnetic Materials, Vol. 259 of NATO Advanced Study Institute, Series B: Physics, edited by G. C. Hadjipanayis and G. A. Prinz (Plenum, New York, 1991).

${ }^{36}$ P.-A. Lindgård, Phys. Rev. B 27, 2980 (1983).

${ }^{37}$ M. N. Barber, in Phase Transitions and Critical Phenomena, edited by C. Domb and J. L. Lebowitz (Academic, New York, 1983), Vol. 8, p. 145.

${ }^{38}$ M. Lottermoser, Z. Angew. Phys. 26, 135 (1969).

${ }^{39}$ D. C. Douglass, J. P. Bucher, and L. A. Bloomfield, Phys. Rev. Lett. 68, 1774 (1992).

${ }^{40}$ Z. X. Tang, C. M. Sorensen, K. J. Klabunde, and G. C. Hadjipanayis, Phys. Rev. Lett. 67, 3603 (1991).

${ }^{41}$ P. J. van der Zaag, A. Noordermeer, M. T. Johnson, and P. F. Bongers, Phys. Rev. Lett. 68, 3112 (1992); V. A. M. Brabers, ibid. 68, 3113 (1992).

${ }^{42}$ J. Jing, X. Yang, Y. Hsia, and U. Gonser, Surf. Sci. 233, 351 (1990).

${ }^{43}$ U. Herr, J. Ring, R. Birringer, U. Gonser, and H. Gleiter, Appl. Phys. Lett. 50, 472 (1987).

${ }^{44} \mathrm{~S}$. Mørup, A. Dumesic, and H. Topsøe, in Applications of Mössbauer Spectroscopy, edited by R. L. Cohen (Academic, New York, 1980), Vol. 2, p. 1.

${ }^{45}$ H. Thomas, Z. Angew. Phys. 15, 201 (1963). 